\title{
Prevalência de olho seco em portadores de hanseníase de um hospital-colônia em Goiânia
}

\author{
Prevalence of dry eye in Hansen's disease patients from a colony hospital in Goiânia, \\ Brazil
}

Karys Carvalho Frazão ${ }^{1}$

Naira Bernardes Picolo ${ }^{2}$

Rosana Zacarias Hanouche ${ }^{3}$

Procópio Miǵuel dos Santos ${ }^{4}$

Regina Cândido Ribeiro dos Santos ${ }^{5}$

Trabalho realizado no Hospital de Dermatologia Sanitária de Goiânia-GO e na Fundação Banco de Olhos de Goiânia (GO).

${ }^{1}$ Mestre em Ciências da Saúde pela Universidade de Brasília (UnB). Brasília (DF).

${ }^{2}$ Médica oftalmologista da Fundação Banco de Olhos de Goiânia. Goiânia (GO).

${ }^{3}$ Médica oftalmologista da Fundação Banco de Olhos de Goiânia. Goiânia (GO).

${ }^{4}$ Professor do Departamento de Oftalmologia do Hospital de Base de Brasília, Doutor em Oftalmologia pela Universidade Federal de São Paulo (UNIFESP). São Paulo (SP).

Professora do Departamento de Oftalmologia do Hospital de Base de Brasília, Doutora em Oftalmologia pela UNIFESP. São Paulo (SP)

Endereço para correspondência: Karys Carvalho Frazão - Av. Dr. Ismerino Soares de Carvalho, 816 -

Goiânia (GO) CEP 74075-040

E-mail: kacaf@hotmail.com

Recebido para publicação em 12.05.2004

Versão revisada recebida em 03.01.2005

Aprovação em 18.03.2005

Nota Editorial: Após concluída a análise do artigo sob sigilo editorial e com a anuência do Dr. Haroldo Vieira

de Moraes Jr. sobre a divulgação de seu nome como revisor, agradecemos sua participação neste processo.

\begin{tabular}{|c|}
\hline RESUMO \\
\hline $\begin{array}{l}\text { Objetivo: Determinar a prevalência de olho seco em hansenianos do } \\
\text { Hospital de Dermatologia Sanitária de Goiânia. Métodos: A amostra do } \\
\text { presente estudo incluiu } 70 \text { hansenianos, do Hospital de Dermatologia } \\
\text { Sanitária de Goiânia, e } 30 \text { controles, da Fundação Banco de Olhos de Goiás, } \\
\text { ambos localizados em Goiânia-GO. Foram realizados exame oftalmológico } \\
\text { e testes de Schirmer I, "break-up time" (BUT) e rosa bengala em todos estes } \\
\text { indivíduos em uma única avaliação. Para o diagnóstico de olho seco foi } \\
\text { considerado o teste de Schirmer I menor ou igual a } 5 \text { mm e o rosa bengala } \\
\text { grau II ou III, em pelo menos um olho. Resultados: Quarenta e quatro } \\
\text { (63,0\%) hansenianos eram do sexo masculino e } 22 \text { ( } 73,3 \%) \text { controles, do } \\
\text { sexo feminino (p=0,001). A idade média dos hansenianos foi de } 61,1 \pm 12,5 \\
\text { anos e no grupo controle, } 55,7 \pm 9,6 \text { anos. Quinze ( } 21,4 \%) \text { hansenianos e } \\
\text { quatro (13,3\%) controles apresentaram diagnóstico de olho seco (p=0,429). } \\
\text { A forma virchowiana (HV) ( } 74,2 \%) \text { da hanseníase foi a mais prevalente e } \\
\text { o olho seco (66,7\%) foi mais freqüente nesta forma clínica da doença. } \\
\text { Conclusão: A prevalência de olho seco foi maior no grupo de hansenianos, } \\
\text { apesar de não ter havido diferença significativa. }\end{array}$ \\
\hline
\end{tabular}

Descritores: Olho seco/hanseníase/forma clínica

\section{INTRODUÇÃO}

A hanseníase, doença infecto-contagiosa e crônica do homem, possui manifestações dermatológicas e neurológicas. O olho pode ser acometido em $6 \%$ a $90 \%$ dos hansenianos, e resulta de lesão direta pelo bacilo, do estado reacional ou pode ser neurogênica ${ }^{(1-2)}$. As alterações oculares da hanseníase que podem levar à cegueira incluem: uveíte crônica, catarata e lagoftalmo com hipoestesia corneana ${ }^{(2-3)}$.

O olho seco na hanseníase pode ser atribuído à ocorrência de lagoftalmo, ectrópio, triquíase e/ou baixa produção de lágrima. Se não for tratado, poderá reduzir a acuidade visual, por úlcera e opacidade da córnea ${ }^{(4-5)}$. Atualmente, a ceratoconjuntivite sicca, ou doença do olho seco, classificase em: deficiência da camada aquosa lacrimal, com hipossecreção aquosa; e/ou evaporativa, com evaporação em excesso, por deficiência da camada lipídica ${ }^{(6-7)}$. Esta é a doença mais freqüente em oftalmologia em todo o mundo, daí a importância do seu estudo. O diagnóstico do olho seco constitui um desafio para o oftalmologista porque os testes não são específicos $^{(8)}$. Os testes para esse diagnóstico avaliam a estabilidade do filme lacrimal (BUT), a camada aquosa e a troca lacrimal (teste de Schirmer, clearance de fluoresceína, teste do fenol vermelho e fluorofotometria), 
alteração da superfície ocular (corantes como o rosa bengala e citologia de impressão) e análise qualitativa do filme lacrimal (osmolaridade e análise de proteínas) ${ }^{(9)}$. Sua definição inclui sintomas inespecíficos, dano à superfície ocular, instabilidade lacrimal e hiperosmolaridade lacrimal ${ }^{(7,10)}$. Para otimizar o diagnóstico de olho seco são necessários vários testes, além de um questionário de sintomas ${ }^{(6,11-12)}$.

O presente estudo teve por objetivo avaliar a prevalência de olho seco em hansenianos, em um hospital-colônia, através dos testes de Schirmer I, do rosa bengala e do tempo de rotura do filme lacrimal (BUT), que são os mais utilizados para medir a função lacrimal ${ }^{(6-7,11)}$.

\section{MÉTODOS}

A presente pesquisa foi aprovada pelo Comitê de Ética em Pesquisa da Faculdade de Ciências da Saúde da UnB com o registro 011/2002. Os prontuários de pacientes do Hospital de Dermatologia Sanitária de Goiânia (HDS) foram revisados e 156 indivíduos com diagnóstico clínico e/ou laboratorial de hanseníase e com a forma clínica da doença especificada em prontuário foram selecionados, com exclusão de indivíduos que se recusaram a participar da pesquisa ou incapazes de se deslocar para o local do exame.

Os controles eram pacientes da Fundação Banco de Olhos de Goiás (FuBOG), não portadores de hanseníase, desprovidos de qualquer patologia ocular e/ou sistêmica que comprometa o filme lacrimal e com idade igual ou superior a 40 anos.

Neste estudo obteve-se consentimento livre e esclarecido de 70 hansenianos (139 olhos) e 30 controles (60 olhos), após exposição sobre a forma de obtenção dos dados e das conseqüências do estudo.

Todos os indivíduos foram submetidos a uma avaliação oftalmológica única, do segmento anterior e de anexos do bulbo ocular, por apenas um examinador, e aos testes de rosa bengala, Schirmer I e BUT para pesquisa de olho seco. O teste de Schirmer I foi realizado primeiro, seguido do BUT e, por último, foi efetuado o teste do rosa bengala, em uma avaliação única, sem intervalo entre os testes.

No teste de Schirmer I foi colocado papel de filtro (Schirmer strips ${ }^{\circledR}$, Ophthalmos, São Paulo-SP) com 35 mm de comprimento e $5 \mathrm{~mm}$ de largura, no fundo de saco conjuntival, em terço lateral da pálpebra inferior do olho. Foi pedido ao paciente para fechar os olhos durante 5 minutos e, após este tempo, foi efetuada a leitura do resultado. Valores inferiores ou iguais a $5 \mathrm{~mm}$ foram considerados positivos; de 6 a $10 \mathrm{~mm}$, suspeitos; e maiores do que $10 \mathrm{~mm}$, negativos ${ }^{(13)}$.

O BUT foi avaliado através do tempo, em segundos, decorrido entre o último piscar e o aparecimento do primeiro ponto negro na córnea após a aplicação de colírio de fluoresceína a $2 \%$ (Fluoresceína $^{\circledR}$, Allergan, São Paulo-SP), observado com luz de cobalto na lâmpada de fenda. Foram feitas três avaliações sucessivas e calculada sua média. $\mathrm{O}$ valor até 10 segundos foi definido como positivo e acima de 10 segundos, negativo ${ }^{(9)}$.

Após a aplicação do colírio de rosa bengala a 1\% (Rosa bengala ${ }^{\circledR}$, Ophthalmos, São Paulo-SP) avaliou-se a coloração da superfície ocular (conjuntiva nasal, temporal e córnea) através de uma fenda larga de baixa intensidade de luz pela biomicroscopia. Foram considerados: grau 0, quando não houve coloração; grau I, quando corou a conjuntiva bulbar; grau II, quando corou a conjuntiva bulbar e o limbo; e grau III, quando corou a conjuntiva bulbar e a córnea, além do limbo ${ }^{(13)}$. Não foi avaliada a intensidade da impregnação do corante.

Para o diagnóstico de olho seco foi considerado o teste de Schirmer I menor ou igual a $5 \mathrm{~mm}$ e o rosa bengala grau II ou III em pelo menos um olho. O teste do BUT não foi utilizado como critério diagnóstico de olho seco.

Para o cálculo estatístico foram utilizados a análise de variância, o teste do qui-quadrado e o teste Exato de Fisher ${ }^{(14)}$ no nível de 5\% de significância. Para a execução dos cálculos matemáticos na análise estatística dos dados foram utilizados os programas Excel e SPSS 8.0.

\section{RESULTADOS}

A amostra do presente estudo incluiu 70 pacientes no grupo de hansenianos $(\mathrm{GH})$ e 30 no grupo controle (GC). No $\mathrm{GH}$, a maior prevalência foi do sexo masculino $(62,9 \%)$ e no GC, do feminino $(73,3 \%)$, havendo diferença entre os dois grupos estudados no que se refere ao sexo $(\mathrm{p}=0,001)$. A idade média no GH foi de $61,1 \pm 12,5$ anos e no GC, de 55,7 $\pm 9,6$ anos, apresentando diferença estatisticamente significativa $(\mathrm{p}=0,037)$ entre os dois grupos, mas não quanto à idade entre os sexos feminino $(\mathrm{p}=0,382)$ e masculino $(\mathrm{p}=0,131)$ nos dois grupos.

Os resultados dos testes de Schirmer I (Tabela 1), do rosa bengala (Tabela 2) e do BUT (Tabela 3) nos indivíduos dos dois grupos estudados foram considerados para cada olho, direito (OD) e esquerdo (OE), separadamente. O teste de Schirmer I foi menor ou igual a $5 \mathrm{~mm}$ em 36 olhos, sendo $22(31,4 \%)$ no OD e 14 (20,3\%) no OE no GH (Tabela 1) e nos indivíduos do GC foi menor ou igual a $5 \mathrm{~mm}$ em 15 olhos, sendo $8(26,7 \%)$ no OD e $7(23,3 \%)$ no OE (Tabela 1). Este teste foi menor do que $11 \mathrm{~mm}$ para a maioria dos indivíduos do $\mathrm{GH}(58,57 \%)$ e do $\operatorname{GC}(53,33 \%)\left(\chi^{2}=0,065, \mathrm{p}=0,798\right)$.

No teste do rosa bengala, os graus II e III constituíram a maioria dos casos dos ODs (51,4\%-36 olhos) e OEs (50,7\%-35 olhos) dos indivíduos do GH (Tabela 2). No GH o grau III ocorreu em $24,3 \%$ ( 17 olhos) de ODs e em $21,7 \%$ ( 15 olhos) de OEs. No GC, o grau II ocorreu em 20,0\% (6 olhos) dos ODs e em $26,7 \%$ ( 8 olhos) dos OEs; o grau III ocorreu em 3,3\% (1 olho) dos OEs e em nenhum OD (Tabela 2). Houve uma maior diferença nos achados entre os dois grupos no que se referiu ao grau III do rosa bengala.

No teste do BUT, a maioria dos indivíduos do GH $(95,7 \%$ de ODs, 67 olhos; e 88,4\% de OEs, 61 olhos) e do GC (96,7\% de ODs, 29 olhos e $100 \%$ de OEs, 30 olhos) apresentou o resultado do teste de até 10 segundos (Tabela 3).

Na tabela 4 verifica-se a relação do olho seco com a idade e o sexo. A freqüência de olho seco no GH foi maior em indivíduos acima de 50 anos, de ambos os sexos $(77,8 \%$ dos homens 


\begin{tabular}{|c|c|c|c|c|c|c|c|c|}
\hline \multirow{3}{*}{$\begin{array}{l}\text { Teste de } \\
\text { Schirmer I } \\
(\mathrm{mm})\end{array}$} & \multicolumn{4}{|c|}{ Grupo de hansenianos } & \multicolumn{4}{|c|}{ Grupo controle } \\
\hline & \multicolumn{2}{|c|}{ OD } & \multicolumn{2}{|c|}{ OE } & \multicolumn{2}{|c|}{ OD } & \multicolumn{2}{|c|}{ OE } \\
\hline & no abs. & $\%$ & no abs. & $\%$ & no abs. & $\%$ & no abs. & $\%$ \\
\hline 0 a 5 & 22 & 31,4 & 14 & 20,3 & 8 & 26,7 & 7 & 23,3 \\
\hline 6 a 10 & 12 & 17,1 & 15 & 21,7 & 8 & 26,7 & 5 & 16,7 \\
\hline$>10$ & 36 & 51,4 & 40 & 58,0 & 14 & 46,7 & 18 & 60,0 \\
\hline Total & 70 & 100,0 & 69 & 100,0 & 30 & 100,0 & 30 & 100,0 \\
\hline
\end{tabular}

\begin{tabular}{|c|c|c|c|c|c|c|c|c|}
\hline \multirow{3}{*}{$\begin{array}{l}\text { Teste do } \\
\text { rosa bengala } \\
\text { (grau) }\end{array}$} & \multicolumn{4}{|c|}{ Grupo de hansenianos } & \multicolumn{4}{|c|}{ Grupo controle } \\
\hline & \multicolumn{2}{|c|}{ OD } & \multicolumn{2}{|c|}{$\mathrm{OE}$} & \multicolumn{2}{|c|}{ OD } & \multicolumn{2}{|c|}{$\mathrm{OE}$} \\
\hline & no abs. & $\%$ & no abs. & $\%$ & no abs. & $\%$ & no abs. & $\%$ \\
\hline 0 & 23 & 32,9 & 19 & 27,5 & 10 & 33,3 & 11 & 36,7 \\
\hline I & 11 & 15,7 & 15 & 21,7 & 14 & 46,7 & 10 & 33,3 \\
\hline II & 19 & 27,1 & 20 & 29,0 & 6 & 20,0 & 8 & 26,7 \\
\hline III & 17 & 24,3 & 15 & 21,7 & - & 0,0 & 1 & 3,3 \\
\hline Total & 70 & 100,0 & 69 & 100,0 & 30 & 100,0 & 30 & 100,0 \\
\hline
\end{tabular}

\begin{tabular}{|c|c|c|c|c|c|c|c|c|}
\hline \multirow{3}{*}{$\begin{array}{l}\text { Teste do } \\
\text { BUT } \\
\text { (seg) }\end{array}$} & \multicolumn{4}{|c|}{ Grupo de hansenianos } & \multicolumn{4}{|c|}{ Grupo controle } \\
\hline & \multicolumn{2}{|c|}{ OD } & \multicolumn{2}{|c|}{$\mathrm{OE}$} & \multicolumn{2}{|c|}{ OD } & \multicolumn{2}{|c|}{$\mathrm{OE}$} \\
\hline & no abs. & $\%$ & no abs. & $\%$ & no abs. & $\%$ & no abs. & $\%$ \\
\hline Até 10 & 67 & 95,7 & 61 & 88,4 & 29 & 96,7 & 30 & 100,0 \\
\hline$>10$ & 3 & 4,3 & 7 & 10,1 & 1 & 3,3 & - & 0,0 \\
\hline Inviável & - & 0,0 & 1 & 1,4 & - & 0,0 & - & 0,0 \\
\hline Total & 70 & 100,0 & 69 & 100,0 & 30 & 100,0 & 30 & 100,0 \\
\hline
\end{tabular}

\begin{tabular}{|c|c|c|c|c|c|c|}
\hline \multirow{2}{*}{$\begin{array}{l}\text { Idade } \\
\text { (anos) }\end{array}$} & \multicolumn{3}{|c|}{ Grupo de hansenianos } & \multicolumn{3}{|c|}{ Grupo controle } \\
\hline & Masculino $^{1}$ & Feminino $^{2}$ & Total $^{3}$ & Masculino $^{1}$ & Feminino $^{2}$ & Total $^{3}$ \\
\hline$<40$ & $1 \quad(11,1)$ & - $\quad(0,0)$ & $1 \quad(6,7)$ & - & - $\quad(0,0)$ & - $\quad(0,0)$ \\
\hline $40-50$ & $1(11,1)$ & - $\quad(0,0)$ & $1 \quad(6,7)$ & - & $2(50,0)$ & $2(50,0)$ \\
\hline $51-60$ & $3(33,3)$ & $2(33,3)$ & $5 \quad(33,3)$ & - & - $\quad(0,0)$ & - $\quad(0,0)$ \\
\hline$>60$ & $4 \quad(44,5)$ & $4 \quad(66,7)$ & $8(53,3)$ & - & $2(50,0)$ & $2(50,0)$ \\
\hline Total & $9(100,0)$ & $6(100,0)$ & $15(100,0)$ & - & $4(100,0)$ & $4(100,0)$ \\
\hline
\end{tabular}

e 100,0\% das mulheres); nos indivíduos do GC ocorreu apenas nas mulheres, em igual freqüência para os indivíduos nas categorias de 40 a 50 anos $(50,0 \%)$ e maiores de 60 anos (50,0\%). Em ambos os grupos estudados, considerando-se os pacientes com diagnóstico de olho seco, não houve diferença estatisticamente significativa com relação à faixa etária por sexo, masculino $(\mathrm{p}=1,000)$ e feminino $(\mathrm{p}=0,429)$, bem como no grupo como um todo $(\mathrm{p}=0,418)$.

Dentre os hansenianos, a forma $\mathrm{HV}$ foi a mais freqüente $(74,29 \%)$ e o olho seco foi mais freqüente nestes pacientes $(66,7 \%)$.
Segundo critérios já descritos anteriormente, 15 indivíduos do GH $(21,4 \%)$ e quatro do GC $(13,3 \%)$ apresentaram olho $\operatorname{seco}\left(\chi^{2}=0,626, p=0,429\right)$.

\section{DISCUSSÃO}

Neste estudo, no GH houve predomínio do sexo masculino, como descrito em vários estudos realizados no Brasil, nos quais a maior prevalência da hanseníase também foi no sexo masculino (de $58,1 \%$ a $72,1 \%)^{(2,4,15)}$, o que pode ter ocorrido 
devido à maior exposição à doença por parte dos homens ou pela resistência natural das mulheres a $\operatorname{ela}^{(16-17)}$.

Nesta pesquisa, a forma virchowiana (HV) da hanseníase foi a mais prevalente, o que está em concordância com os achados de várias pesquisas com portadores desta patologia em tratamento ambulatorial ${ }^{(2,4-5,15)}$. Em um estudo com hansenianos de um hospital colônia foi encontrada a forma HV da doença em $94,2 \%$ dos pacientes examinados ${ }^{(18)}$, a qual é a mais freqüiente no Brasil, segundo dados da literatura ${ }^{(17)}$. Apesar de a forma HV ser a mais freqüente no Brasil, na África e na Índia prevalece a forma tuberculóide (HT) ${ }^{(17)}$.

Os resultados encontrados nesta pesquisa para os testes Schirmer I, rosa bengala e BUT estão descritos separadamente para OD e OE, nas tabelas 1, 2 e 3, respectivamente, porém a análise deles foi feita por paciente, visto que o acometimento ocular nesta doença sistêmica é bilateral, podendo ser assimétrico. O teste de Schirmer foi empregado, neste estudo, porque é um teste objetivo, que mede a parte aquosa da produção lacrimal, sendo o teste mais utilizado na prática clínica e de fácil execução ${ }^{(7,9,18)}$. Quando só o teste de Schirmer é considerado para o diagnóstico de olho seco, podem ocorrer resultados falso-positivos e negativos ${ }^{(7)}$, pois as condições ambientais e a hora do dia alteram seu resultado ${ }^{(7,9,12)}$. O teste do BUT foi empregado, neste estudo, para avaliação da estabilidade do filme lacrimal, que se encontra reduzida na deficiência da camada de mucina do filme lacrimal, apesar de não ser um teste útil para diagnóstico específico ${ }^{(19-20)}$.

O teste do rosa bengala foi empregado, neste estudo, para avaliar a vitalidade do epitélio da córnea e da conjuntiva, medindo de maneira indireta a secreção lacrimal ${ }^{(7,18,20-21)}$. Nenhum dos estudos com hansenianos utilizou o teste do rosa bengala para o diagnóstico de olho seco, porém este teste é referido como o mais confiável ${ }^{(20)}$. No teste do rosa bengala, o grau III foi mais freqüente no GH do que no GC possivelmente pelo maior comprometimento da superfície ocular nestes olhos, decorrente da falta de lubrificação do olho.

Ao ser considerado somente o teste de Schirmer I para o diagnóstico do olho seco foi encontrado, no presente trabalho, um resultado semelhante entre os pacientes do $\mathrm{GH}$ e do GC $(p=0,798)$, corroborando os resultados de Koshy et al. ${ }^{(22)}$. A prevalência de olho seco avaliada apenas pelo teste de Schirmer alterado, em hansenianos, variou de $9,2 \%$ a $62 \%$ em estudos anteriores ${ }^{(2,5,15-16,23-24)}$, resultados semelhantes aos desta pesquisa. Entretanto, os estudos citados não utilizaram um grupo controle, avaliando a freqüência do olho seco somente nos hansenianos e diferindo quanto aos valores utilizados do teste de Schirmer para definir esta patologia, o que dificulta a comparação entre eles.

Neste estudo, a maioria dos indivíduos do GH e do GC apresentou BUT menor do que 10 segundos (Tabela 3) e as prováveis razões para isto incluem: instabilidade do filme lacrimal, pela reduzida umidade do ar e alta temperatura em Goiânia e/ou pela manutenção da abertura da pálpebra pelo examinador nos pacientes que não conseguiam parar de piscar. O BUT não foi utilizado como critério diagnóstico de olho seco porque seu resultado foi semelhante nos indivíduos de ambos os grupos, contrastando com o estudo de Koshy et al. ${ }^{(22)}$, que encontraram diferenças estatisticamente significativas entre hansenianos e controles. Neste estudo, a maioria dos hansenianos com alterações palpebrais que apresentou BUT reduzido pode ter sido pelo aumento da evaporação da lágrima ${ }^{(22)}$.

$\mathrm{Na}$ presente investigação, o olho seco ocorreu mais na forma clínica $\mathrm{HV}$, o que está em concordância com o estudo de Sekhar et al. ${ }^{(23)}$, porém, outros estudos não encontraram diferença entre as formas multibacilar e paucibacilar ${ }^{(16,22)}$. Isto pode ter ocorrido por ser este o grupo mais prevalente no presente estudo e/ou porque o olho seco pode não ser exclusivamente pela hanseníase, mas devido à idade avançada, ao sexo feminino e/ou ao uso de medicamentos. Deve-se também considerar que o número de casos de olho seco nas diversas formas clínicas foi pequeno para determinar a forma clínica predominante neste estudo. Na literatura também há achados que diferem do presente estudo, pois as alterações intraoculares ocorrem mais na forma $\mathrm{HV}$ e as alterações da porção externa do olho, na forma $\mathrm{HT}^{(25)}$

No presente trabalho, o olho seco foi mais freqüente nas idades mais avançadas no GH e no GC (Tabela 4), o que está de acordo com a literatura ${ }^{(10)}$. A frequiência semelhante de olho seco nos sexos masculino e feminino no GH pode ter sido pela maior prevalência da hanseníase nos homens que, portanto, se aproximaram das mulheres na frequiência do olho seco. Já no GC, a ocorrência do olho seco apenas nas mulheres concorda com a literatura ${ }^{(10)}$. Os diversos estudos que avaliam a freqüência de olho seco em hansenianos não correlacionam o olho seco com a idade ou o sexo.

Os critérios utilizados no presente trabalho, já descritos anteriormente, são mais rígidos do que os de outros estudos e podem ter reduzido o índice de resultados falso-positivos, mas com aumento do número de falso-negativos ${ }^{(13)}$. O olho seco no GH não pôde ser atribuído exclusivamente à doença, pois pode ter ocorrido pelo uso de medicamentos, idade avançada e/ou deficiência hormonal, no sexo feminino, visto que a sua freqüência não diferiu da do $\mathrm{GC}(\mathrm{p}=0,429)$. Os hansenianos excluídos, neste estudo, podem ter induzido vieses na freqüência do olho seco neste grupo.

Para os pacientes em que os testes de Schirmer I ou do rosa bengala foram suspeitos para olho seco são necessários testes adicionais para confirmar ou excluir o diagnóstico desta patologia, exceto naqueles com alterações de anexos oculares que comprometam a superfície ocular e alterem estes testes ${ }^{(5)}$.

Para o diagnóstico do olho seco são necessárias a associação de testes e a correlação com a sintomatologia do paciente ${ }^{(7)}$. Além dos testes tradicionais já mencionados, têm sido relatados novos testes como: osmolaridade do filme lacrimal, radioimunoensaio da proteína do filme lacrimal e clearance da fluoresceína ${ }^{(6,9)}$.

O diagnóstico do olho seco ainda constitui um desafio para os clínicos, por não existir um teste específico ${ }^{(23)}$, havendo necessidade da avaliação quanto à sensibilidade e à especificidade dos diversos testes utilizados na investigação desta patologia em estudos futuros. 


\section{CONCLUSÕES}

O olho seco foi mais prevalente nos hansenianos, apesar de não ter havido diferença estatística significativa em relação aos controles. O olho seco, portanto, não pôde ser atribuído exclusivamente à hanseníase, podendo ter ocorrido pelo uso de medicamentos, idade avançada e/ou alterações hormonais.

\section{ABSTRACT}

Purpose: To determine the prevalence of dry eye in Hansen's disease patients from the "Hospital de Dermatologia Sanitária de Goiânia". Methods: The sample of the present study included 70 patients with Hansen's disease, from the "Hospital de Dermatologia Sanitária de Goiânia", and 30 controls, from the "Fundação Banco de Olhos de Goiás", both located in Goiânia-GO, Brazil. Ophthalmologic examination and Schirmer I, break-up time (BUT) and rose Bengal staining tests were carried out for all patients in a single evaluation. For the diagnosis of dry eye the Schirmer I equal or less than $5 \mathrm{~mm}$ and rose Bengal grade II or III, in at least one eye were considered. Results: Forty-four $(63.0 \%)$ patients with Hansen's disease were male and $22(73.3 \%)$ controls were female $(\mathrm{p}=0.001)$. The mean age of the patients with Hansen's disease was 61.1 \pm 12.5 years and in the control group, it was $55.7 \pm 9.6$ years. Fifteen $(21.4 \%)$ patients with Hansen's disease and four (13.3\%) controls presented with dry eye diagnosis $(\mathrm{p}=0.429)$. The lepromatous type (74.2\%) of Hansen's disease was the most prevalent and dry eye $(66.7 \%)$ was more frequent in this clinical form of the disease. Conclusion: The dry eye disease was more prevalent in patients with Hansen's disease, although the difference was not statistically significant.

Keywords: Dry eye/Hansen's disease/clinical form

\section{REFERÊNCIAS}

1. Campos WR, Rodrigues CAF, Oréfice F. Hanseníase. In: Oréfice F, editor. Uveíte: clínica e cirúrgica: texto \&. atlas. Rio de Janeiro: Cultura Médica; 2000. p.41-66.

2. Toribio RC, Mendes GF, Alvarez RRA, Souza ALB. Alterações oculares e incapacidade visual em pacientes com hanseníase: um estudo no Distrito Federal. An Bras Dermatol. 2001;76(5):543-50.
3. Ffytche TJ. The prevalence of disabling ocular complications of leprosy: a global study. Indian J Leprosy. 1998;70(1):49-59.

4. Oliveira Neto HL, Silva JLM, Manso PG, Botene IM, Sartori MBF. Envolvimento ocular na hanseníase: estudo em pacientes de ambulatório. Arq Bras Oftalmol. 1996;59(2):162-6.

5. Passerotti S, Salotti RA, Vieth H. Assessment and treatment of the dry eye in leprosy. Indian J Leprosy. 1998;70(1):103-8.

6. Pflugfelder SC, Solomon A, Stern ME. The diagnosis and management of dry eye: a twenty-five-year review. Cornea. 2000;19(5):644-9.

7. Bron AJ. Diagnosis of dry eye. Survey Ophthalmol. 2001;45(Suppl 2):S221-6.

8. Lemp MA, Chacko B. Diagnosis and treatment of tear deficiencies. In: Tasman W, Jaeger EA. Duane's clinical ophthalmology. Philadelphia: Lippincott-Raven; 1995. p.1-13.

9. Sato H, Leoratti MCV. Atualidades doenças externas e córnea: olho seco. In: Souza LB, Vieira LA, Santos WD, editores. Atualidades em oftalmologia. São Paulo: DEOC-UNIFESP; 2001. p.1-12.

10. Murube J. Classificação do olho seco. In: Simpósio Olho Seco. São Paulo: Medicöpea, 2000. p.43-7.

11. Pflugfelder SC, Tseng SC, Sanabria O, Kell H, Garcia CG, Felix C, et al. Evaluation of subjective assessments and objective diagnostic tests for diagnosing tear-film disorders known to cause ocular irritation. Córnea. 1998;17(1): 38-56.

12. Pflugfelder SC. Terapia antiinflamatória melhora sinais e sintomas de olhos secos. Ocular Surg News. 2001;3(5):25-6.

13. Mendes LE, Santos PM, Parente FSP, Gonçalves JOR, Belfort Jr. R. Olho seco em pessoas com idade superior a 40 anos selecionadas em três cidades brasileiras. Arq Bras Oftalmol. 1995;58(1):10-5.

14. Monteiro Filho G. Estatística prática para ciências biológicas. Goiânia: Vieira; 2000. v.1.

15. Monteiro LG, Campos WR, Oréfice F, Grossi MA. Study of ocular changes in leprosy patients. Indian J Leprosy. 1998;70(2):197-202.

16. Santos PM, Maradei J, Barra JMM, Santos RCR, Abreu MT. Estudo das alterações oculares em portadores de hanseníase de hospital colônia. Arq Bras Oftalmol. 1995;58(2):130-7.

17. Sampaio SAP, Rivitti EA. Hanseníase. In: Sampaio SAP, Rivitti EA, editores. Dermatologia. São Paulo: Artes Médicas; 1998. p.467-87.

18. Mattar DB, Kara-José N. Filme lacrimal. In: Belfort Jr. R, Kara-José N. Córnea clínica-cirúrgica. São Paulo: Roca; 1996. p.33-40.

19. Laibson PR. Diagnosis and treatment of keratoconjunctivitis sicca. In: Barraquer JI, Binder PS, Buxton JN, Fine M, Jones DB, Laibson PR, et al. Symposium on medical and surgical diseases of the cornea: transactions of the New Orleans Academy of Ophthalmology. St. Louis: The C.V. Mosby; 1980. p.36-47.

20. Scarpi MJ. Olho seco. In: Belfort Jr R, Kara-José N, editores. Córnea clínicacirúrgica. São Paulo: Roca; 1996. p.285-99.

21. Yamane R, Câmara AG. Filme lacrimal, testes de secreção e drenagem lacrimal e estesiometria. In: Yamane R. Semiologia ocular. Rio de Janeiro: Cultura Médica; 1990. p.103-8. (Biblioteca Brasileira de Oftalmologia).

22. Koshy S, Daniel E, Kurian N, Yovan P. Pathogenesis of dry eye in leprosy and tear functions. Intern J Lepr Other Mycobact Dis. 2001;69(3):215-8.

23. Sekhar GC, Vance G, Otton S, Kumar SV, Stanley JN, Rao GN. Ocular manifestations of Hansen's disease. Doc Ophthalmol. 1994;8(3):211-21.

24. Colodetti SCZ, Colodetti LSD, Moraes Jr. HV. Estudo das alterações oculares em pacientes hansenianos provenientes de área hiperendêmica (Município de Sooretama, Espírito Santo). Rev Bras Oftalmol. 2003;62(7):516-23.

25. Dana MR, Hochman MA, Viana MAG, Hill CH, Sugar J. Ocular manifestations of leprosy in a noninstitutionalized community in the United States. Arch Ophthalmol. 1994;112:626-9.

\section{Nos artigos enviados para publicação, o nome dos autores e suas afiliações devem estar completos. Isso facilitará a indexação e os links com as bases de dados e o CV Lates.}

